Erratum

\title{
Erratum: Mahgoub et al., Effect of Carbamazepine and Sodium Valproate on Liver Enzymes of Epileptic Children. Dr. Sulaiman Al-Habib Medical Journal. Vol. 2(3); September (2020), pp. 123-127
}

\author{
Abdelmoneim Mahgoub ${ }^{1}$, M. Abdoun ${ }^{1, *},(\mathbb{D})$, Sohail Azam², (D), Reham Babiker ${ }^{1}$ \\ ${ }^{1}$ Department of Pediatric, Arryan Hospital, Dr Sulaiman Al Habib Medical Group, Khurais Road, P.O. Box 100266, Riyadh 11635, Saudi Arabia
} ${ }^{2}$ Department of Inpatient Pharmacy, Arryan Hospital, Dr Sulaiman Al Habib Medical Group, Riyadh, Saudi Arabia

A word "ALT" has been changed inadvertently to "AST" in Table 7 during the production process and the same is published online [https://doi.org/10.2991/dsahmj.k.200902.001]. Corrected Table 7 reads as follows:

Table 7 Characteristics of the study population based on ALT level ${ }^{\mathrm{a}}$

\begin{tabular}{lccccc}
\hline \multirow{2}{*}{ ALT } & & \multicolumn{2}{c}{ Carbamazepine } & & \multicolumn{2}{c}{ Sodium valproate } \\
\cline { 6 - 6 } \cline { 5 - 6 } \cline { 5 - 6 } Variable & Normal & Altered & & Normal & Altered \\
\hline Age & $7 \pm 3.15$ & $5.6 \pm 3.7^{*}$ & & $7.74 \pm 4.20$ & $11.0 \pm 6.92^{*}$ \\
Male & 25 & $1^{*}$ & & 28 & $3^{*}$ \\
Female & 22 & 2 & & 19 & 0 \\
ALT (10-40 IU/L) & $14.93 \pm 6.5$ & $39.3 \pm 0.57^{* *}$ & & $14.40 \pm 6.43$ & $48.0 \pm 10.58^{* *}$ \\
$\quad$ Mean \pm SD & & & & \\
a & & & &
\end{tabular}

